\title{
LOST OSCILLATIONS: EXPLORING A CITY'S SPACE AND TIME WITH AN INTERACTIVE AUDITORY ART INSTALLATION
}

\author{
Jim Murphy, Dugal McKinnon, Mo H. Zareei \\ New Zealand School of Music, Victoria University of Wellington \\ PO Box 600, Wellington 6140, New Zealand \\ Jim.Murphy@vuw.ac.nz, Dugal.McKinnon@vuw.ac.nz, Mo.Zareei@vuw.ac.nz
}

\begin{abstract}
Lost Oscillations is a spatio-temporal sound art installation that allows users to explore the past and present of a city's soundscape. Participants are positioned in the center of an octophonic speaker array; situated in the middle of the array is a touch-sensitive user interface. The user interface is a stylized representation of a map of Christchurch, New Zealand, with electrodes placed throughout the map. Upon touching an electrode, one of many sound recordings made at the electrode's real-world location is chosen and played; users must stay in contact with the electrodes in order for the sounds to continue playing, requiring commitment from users in order to explore the soundscape. The sound recordings have been chosen to represent Christchurch's development throughout its history, allowing participants to explore the evolution of the city from the early 20th Century through to its post-earthquake reconstruction. This paper discusses the motivations for Lost Oscillations before presenting the installation's design, development, and presentation.
\end{abstract}

\section{INTRODUCTION AND MOTIVATIONS}

Sound archives allow dedicated researchers access primary sources associated with the history of a place and the events which shaped it. By engaging in a longitudinal listening survey, as a kind of sonic archeology, researchers may unearth the development of a place, but also vicariously experience significant events that have affected an area over the course of its (phonographic) history. Those persons less able or inclined to peruse large amounts of recorded material, they may be largely unaware of the sonic stratigraphy upon which they live. One use of auditory display in an artistic installation context is to convey this sonic history to the public in an affectively compelling and aesthetically-motivated manner.

This paper explores one such sonic artwork, the interactive Lost Oscillations installation. Installed in Christchurch, New Zealand in October, 2015, Lost Oscillations allows participants to engage in a multi-sensory exploration of recorded events throughout the last eight decades of Christchurch's history.

In informal contemporary dialog, Christchuch's history prior to the 2010 and 2011 Canterbury earthquakes is muted: the earthquakes function as a sort of 'event horizon,' and there is little disCommercial 4.0 International License. The full terms of the License are available at http://creativecommons.org/licenses/by-nc/4.0

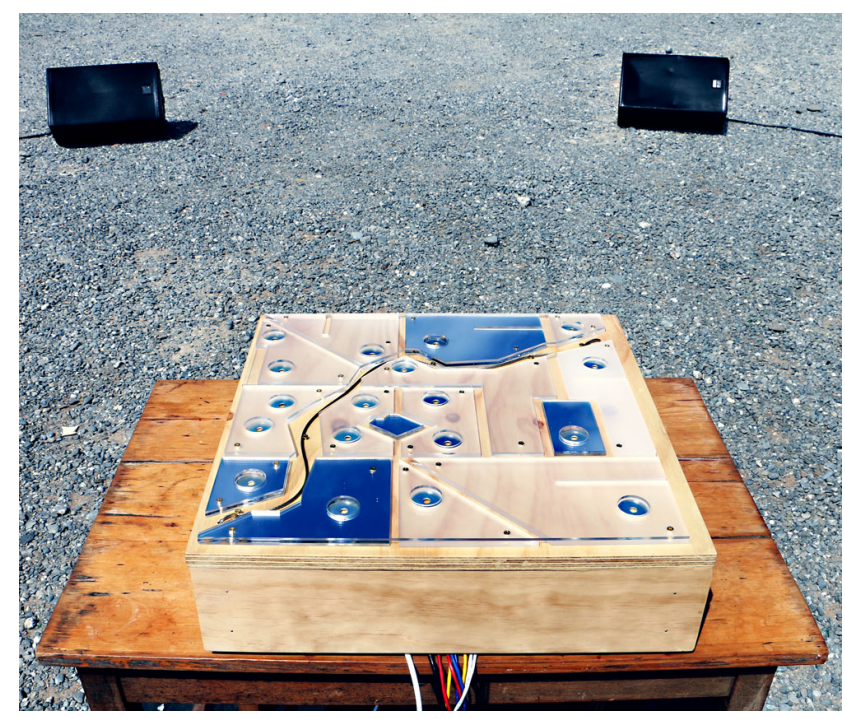

Figure 1: The purpose-built Lost Oscillations user interface, shown with two of its eight loudspeakers.

cussion of events preceding them. A key goal of Lost Oscillations is to slip past these traumatic events, allowing those interacting with the artwork to engage, through touch and listening, with Christchurch in a context that largely focuses on its pre-earthquake history. A tactile, multi-user interactive interface (shown in Figure 1) was chosen as the means by which participants may engage with an array of archival recordings. This decision was arrived at both to allow communal experience of sonic history and to emphasize embodied connection, through sound and touch, to sonic place. To further provide those visiting the artwork with an immersive, mixed reality, site-specific auditory experience, sounds triggered from the interface are output to an eight-channel loudspeaker array. This octophonic array spatializes the sounds relative to the installation's central-Christchurch location: sounds recorded in locations to the north of the installation space, for example, are output to northward-located loudspeakers.

The remainder of this paper provides a technical and aesthetic overview of Lost Oscillations. It begins with a discussion of related works, presenting a number of pieces whose usage of interface design and artistic aesthetic were influential in the artwork's initial conception and development. Following the review of related works, an overview of the design, development, and construction of Lost Oscillations is provided. Section 3.3 discusses 
the purpose-built interface, its connection to a host computer, and the computer's audio output configuration. Similarly, Section 3.4 details the user interface's microcontroller firmware as well as the audio playback software used on the audio host PC. Finally, Section 3.2 discusses the audio used in Lost Oscillations, including both archival recordings and contemporary field recordings. After discussing the physical design of the artwork, the installation and use of Lost Oscillations is presented, focusing on its interactivity and engagement by the public. Finally, the paper concludes with a discussion of future avenues for similar works as well as a number of potential improvements that may be undertaken in future iterations of Lost Oscillations.

\section{RELATED WORKS}

Lost Oscillations fuses three sonic arts subdisciplines together, including elements pertaining to the development of new interfaces for musical expression, spatio-temporal sound art, and acousmatic composition approaches. As such, key works from each of the three subdisciplines served as inspirations for Lost Oscillations. This section details a number of such works.

\subsection{Interface Design and Diffusion Performance}

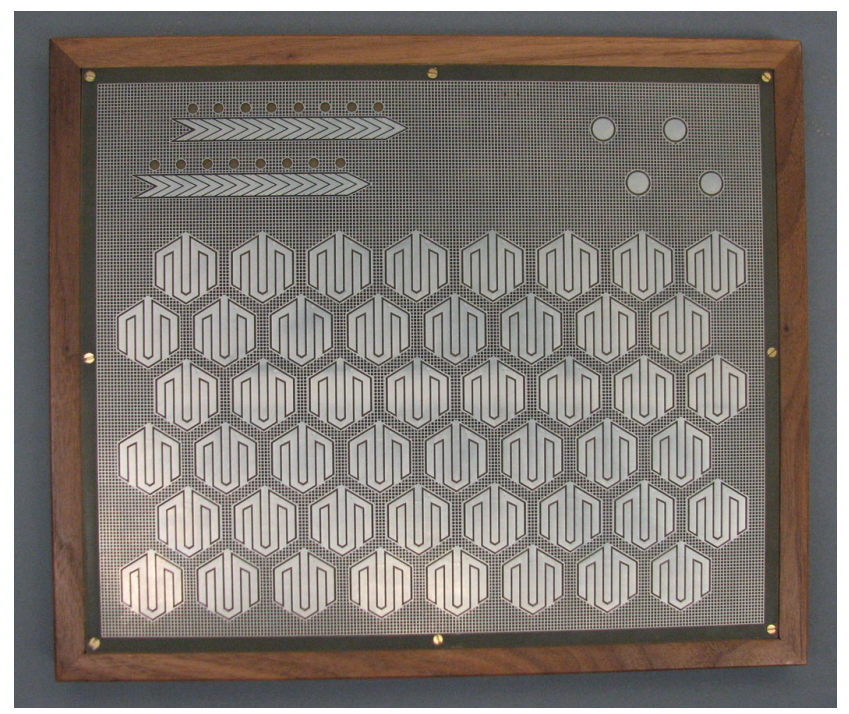

Figure 2: The Manta touch-sensitive audio interface: an array of touch-sensitive electrodes that may be configured for audio interfacing applications. Photo courtesy http://www.snyderphonics.com/

There is a rich history of new musical controller interfaces featuring touch-sensitive electrodes. Whether capacitive or resistive, these interfaces allow a user to affect an auditory output through the use of a touch event. An early example of such interfaces is the Cracklebox, a small analog soundmaker module developed in 1973 by Michel Waisvisz at STEIM ${ }^{1}$. The Cracklebox's influence has extended to modern MIDI-enabled musical performance tools, including the recent Snyderphonics Manta ${ }^{2}$. The Manta (shown in

\footnotetext{
${ }^{1}$ http://steim.org/product/cracklebox/

${ }^{2}$ www.snyderphonics.com/manta.htm
}

Figure 2) is a touch-sensitive MIDI controller whose configuration was a key influence during the design of the Lost Oscillations interface: capable of USB-based MIDI communication, the Manta may be configured to trigger and affect audio events on a PC.

Further, the second author's prior audio interfaces were also used as reference points in the design of the Lost Oscillations interface: the decision to pursue touch input with no tactile feedback was chosen in part due to the corporeal engagement afforded by the membrane potentiometer-based Helio interface, described in [1].

In addition to turning to notable general purpose performance interfaces for inspiration when developing Lost Oscillations, a number of multichannel-specific interfaces were examined. [2] provides a history of such interfaces, many of which are used for live 'diffusion' performance and feature audio mixing desk-style user interaction schemes, containing arrays of linear potentiometers for adjusting loudspeaker gains. In contrast to these generalpurpose interfaces, intended to be used across a number of different music genres, the Lost Oscillations interface allows users to explore a predefined range of samples. As such, a sample-set specific interface may be used, allowing for a close coupling between interface and audio.

\subsection{Spatio-Temporal Sound Art}

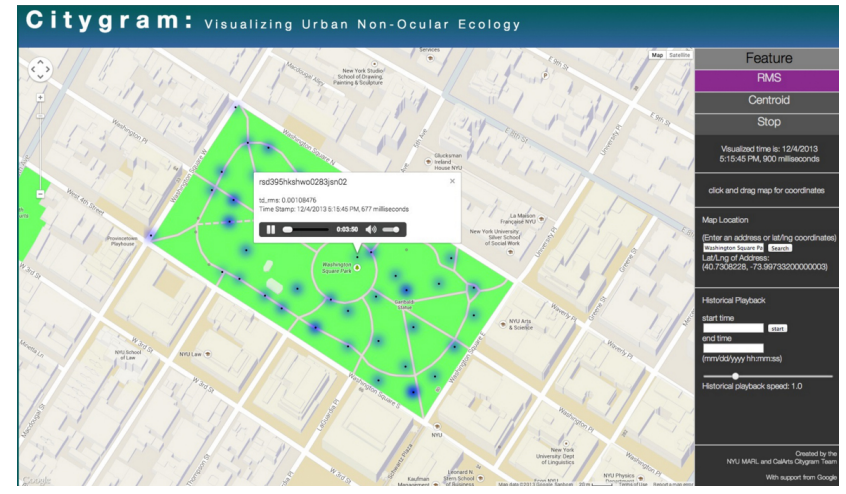

Figure 3: The Citygram interface, showing a map with audio features spatially illustrated. Screenshot courtesy http://cds.nyu.edu/projects/citygram-sound/

In developing Lost Oscillations, existing artworks that allow participants to explore the spatiotemporality of a city or place were examined. Third author Mo H. Zareei's Complex, a "physical resonification of urban noise" (described in detail in [3]) was a key prior inspiration to Lost Oscillations: with a number of kinetic sound sculptures positioned on a map in locations relative to microphone locations in the city, Zareei's work made use of the Citygram locative sonification dataset (described in [4] and illustrated in Figure 3) to convey a city's temporally-morphing sonic featureset.

Lost Oscillations shares this spatial coupling, using its maplike interface to allow participants to explore sounds recorded at locations upon which electrodes are placed on the interface. Where Complex is a self-running kinetic sound sculpture, Lost Oscillations focuses on participatory engagement with the piece, requiring people to engage with the interface to remain in physical contact with it in order for audio elements to be played back. 


\section{DESIGN, DEVELOPMENT, AND USER EXPERIENCE}

The development of Lost Oscillations focused on three areas: the system's hardware, its software, and the accompanying audio files to be played back. After providing an overview of the piece's functioning, this section discusses each of these three and details the means by which participants interact with the artwork.

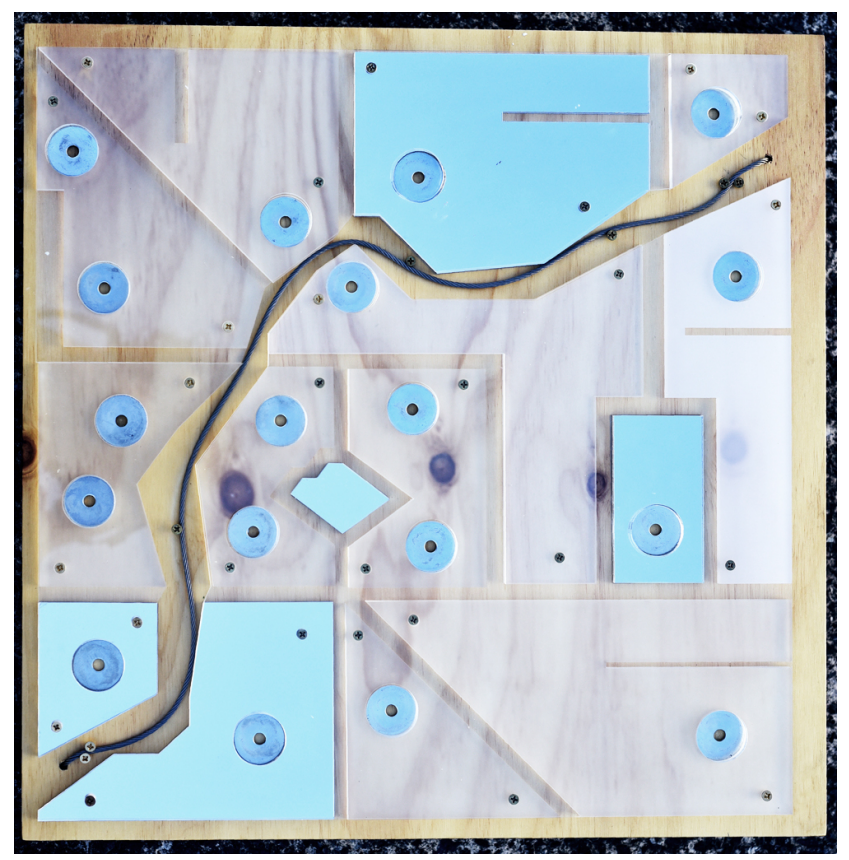

Figure 4: Top view of the Lost Oscillations user interface, showing electrodes placed throughout a map-like representation of the city center of Christchurch, New Zealand.

\subsection{User Experience}

Upon approaching the user interface at the center of a ring of loudspeakers, a participant sees a sculptural interface (shown in Figure 4) made of wood and plastic and interspersed with a number of metal pads. Upon touching the pads, an ambient soundscape produced by the loudspeaker array fades out to be replaced by an audio recording emanating from the loudspeakers in the direction of the sound file's location of recording. The participant may touch more than one metal pad, resulting in multiple playback events, each spatialized relative to their real-world recording location. After moving away from the contact points, the recordings fade out and are slowly replaced by ambient audio emanating from all directions. Another significant aspect of the interaction with audio materials is that users cannot access the same temporal point in any audio file as the audio is constantly, but silently, playing in realtime and only becomes audible when an electrode is touched. here the intention was to highlight time, and in particular the evanescent of sound and the vital role of memory in auditory experience given its invisible, intangible sound objects.

The following subsections provide specific details about the development of the project, with a focus on the audio files used as well as the hardware of the user interface and the software controlling audio playback.

\subsection{Audio}

The development of Lost Oscillations began with the selection and creation of audio files to be played back in response to user input. 110 sound files were selected, spanning the years 1935 to 2015. These sound files were obtained both from Ngā Taonga Sound and Vision, New Zealand's archive of film and video, and also include self-made field recordings that were recorded in Christchurch in the weeks prior to the installation of Lost Oscillations.

The archival audio samples from Ngā Taonga Sound and Vision were chosen to equally represent everyday events and historically-significant events. Further, the audio was selected with consideration given to the location of its recording: by selecting recordings made at the same locations over the course of many decades, a sort of stratigraphic column of sound material is formed. Sitting at the top of this column are the most recent recordings, created by the third author during 2015 in the weeks leading up to the piece's installation. These contemporary field recordings serve to provide a direct coupling between the "realworld" sonic cityscape, in which the participants are immersed, and the recorded soundscapes played through the loudspeaker array and thereby maximize the mixed-reality ambiguity described below.

After selecting the sound files from Ngā Taonga Sound and Vision and creating a number of field recordings, the sound files were organized according to the location of their recording. All of the archival and field recording made in a single location were then merged together into a single audio file, creating a temporal stream of audio anchored to a single point in space.

\subsection{Hardware}
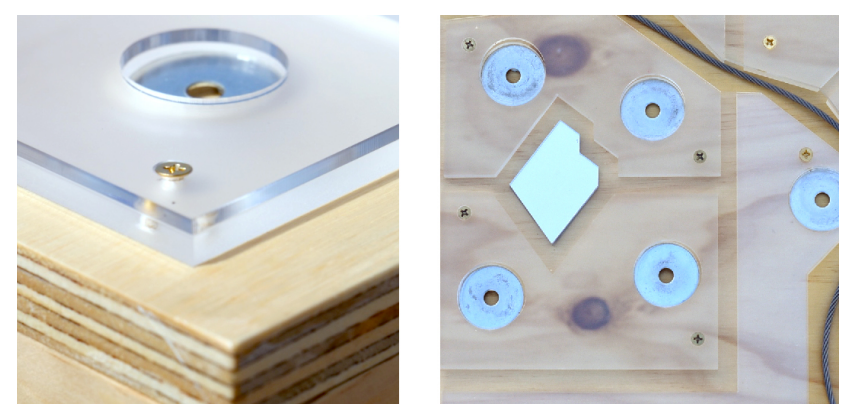

Figure 5: Detailed views of the user interface's electrodes. At left, an electrode mounted within a plastic panel; at right, a number of electrodes made from metal washers and wire braid.

After selecting, organizing, and compiling the sound files into discrete streams, the work on Lost Oscillations shifted to the realization of the user input device and the interfacing schemes between the input device and the audio playback software.

In essence, the user interface in Lost Oscillations is a MIDI input device that transmits MIDI messages in response to user interaction events. Physically, the interface consists of a large wooden box that serves both as enclosure for transducer control and communications electronics and as a mounting chassis for the interface's electrodes.

The purpose-built physical enclosure is constructed from materials chosen as representative of the post-earthquake 
Christchurch rebuild, containing plywood surfaces, plastic ornamentation, and metal electrodes made from wire braid and large washers of the type used in building construction projects (shown at right in Figure 5). These materials were selected with the intention of affording tangible symbolic engagement with the city. Such a focus on the affective properties of materiality (as explored in Jane Bennett's Vibrant Matter [5]) serves to further couple Lost Oscillations to the location in which it is installed. Further, the layers of materials on the interface (shown at left in Figure 5) provide a visual connection to the sort of aural stratigraphic layering present in the audio files (discussed in Section 3.2).

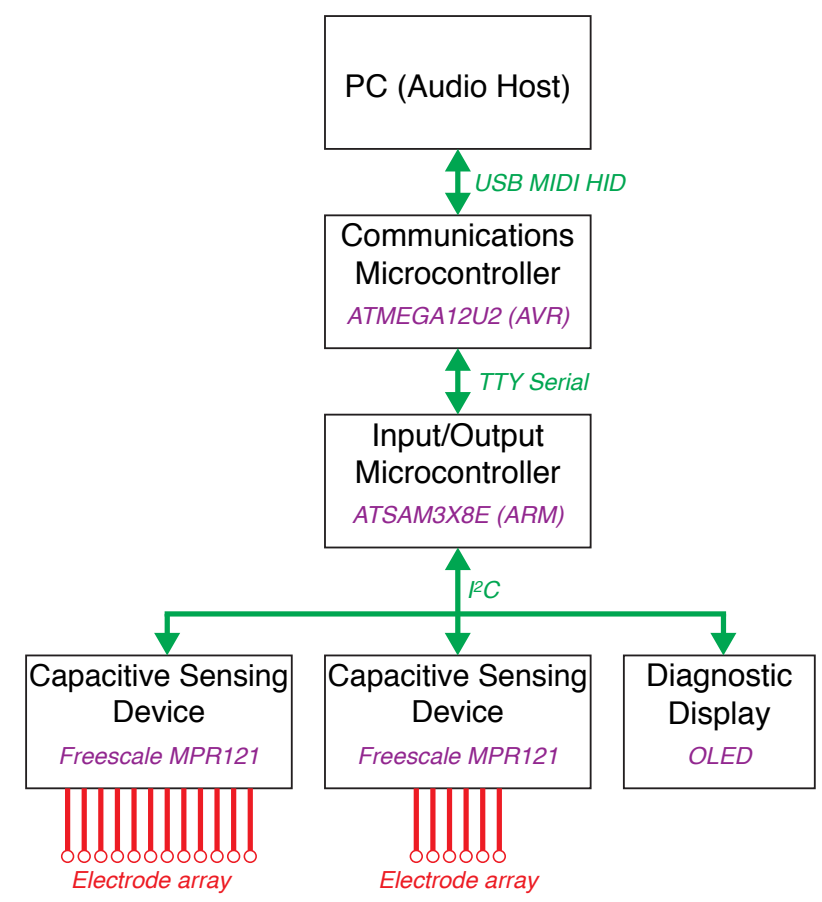

Figure 6: A block diagram illustration the Lost Oscillations user interface circuit.

The Lost Oscillations electronics consist of a number of subassemblies that are interconnected, acting together to allow touches to be detected and result in audio playback on a host PC. Figure 6 shows a block diagram of the electronics, which are described below.

Each of the 19 electrodes is connected to a Freescale MPR121 capacitive sensing device. The MPR121 is intended to allow a number of electrodes to easily be connected to a microcontroller. To simplify communications between a host microcontroller and the capacitive sensing device, the MPR 121 uses the $\mathrm{I}^{2} \mathrm{C}$ protocol (described in more detail by [6]). The $\mathrm{I}^{2} \mathrm{C}$ protocol allows multiple MPR121 devices to be connected together on a shared bus, with each device assigned its own address. As the MPR121 allows for a maximum of 12 electrodes to be connected, a second MPR121 is used in the Lost Oscillations interface to allow for all of the 19 electrodes to be scanned.

The $\mathrm{I}^{2} \mathrm{C}$ bus employed by Lost Oscillations allows for twoway communication: the MPR121 devices may be digitally configured at startup in addition to subsequently reporting their electrode states (discussed below in Section 3.4). This configuration capability allows the electrode sensitivities to be individually set, decreasing the chance of false positive electrode sensing events. In addition to using $\mathrm{I}^{2} \mathrm{C}$ to control and communicate with the MPR121 devices, a small $\mathrm{I}^{2} \mathrm{C}$ OLED diagnostic display is also connected to the bus. This display allows for rapid debugging and status checks to be conducted while the artwork is installed in the field.

The Lost Oscillations user interface employs an Arduino Due microcontroller development board to handle its input, output, and communications. The Arduino Due consists of two separate microcontrollers: the primary microcontroller is a 32-bit ATSAM3X8E ARM device; the secondary microcontroller is an 8-bit ATMEGA32U4 AVR device. The primary microcontroller is used to communicate with the MPR121 capacitive sensors. It communicates with the secondary microcontroller via TTY serial messages. The secondary microcontroller's role is to convert the TTY serial messages from the primary microcontroller into USB MIDI HID messages that may be read by a digital audio workstation on a host PC. To serve as a USB MIDI HID device, the secondary microcontroller is programmed with the HIDUINO firmware, allowing for driverless MIDI communications [7].

The $\mathrm{I}^{2} \mathrm{C}$ devices along with the two microcontrollers make up the purpose-built electronics assembly for the Lost Oscillations user interface. Due to the universal compatibility of the HIDUINO-equipped MIDI HID device, any computer with an operating system designed to handle HID systems may be used for receiving MIDI touch events for Lost Oscillations. In practice, a Mac Mini was chosen due to its relative low cost, small size, and compatibility with the digital audio workstation software discussed in the following subsection.

\subsection{Software}

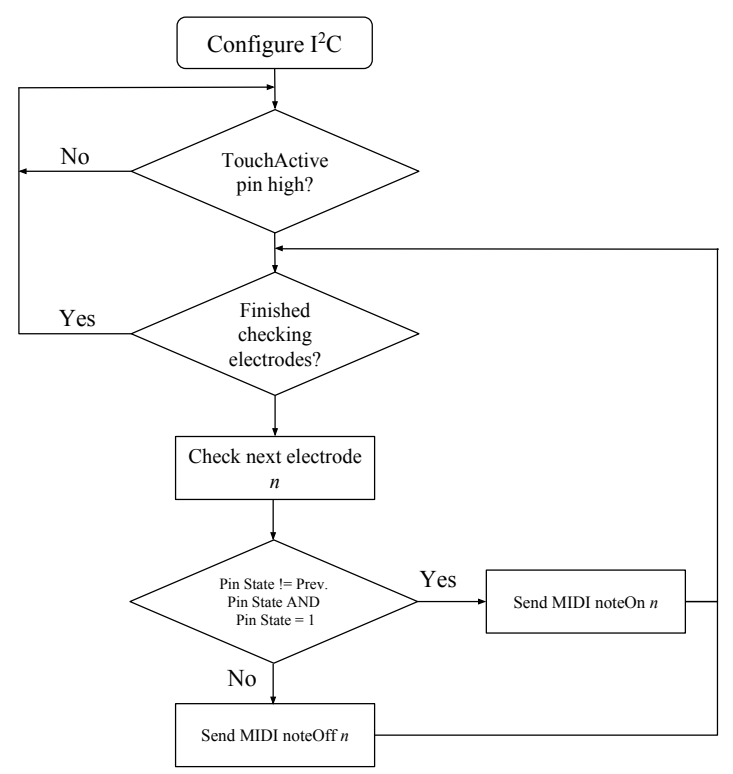

Figure 7: A program flow diagram of the capacitive sensor and MIDI communications scheme deployed on the ATSAM3X8E. 
Lost Oscillations makes use of two software subsystems: one on the microcontroller assembly presented in the preceding subsection and a second on the audio host PC.

As discussed above, the microcontroller assembly consists of two separate microcontrollers, one to handle MIDI and one to handle user input and output. While the MIDI communications microcontroller is equipped with a standard HIDUINO firmware (as presented in [7]), the input/output microcontroller contains a customdeveloped firmware.

Figure 7 is a program flow diagram illustrating the means by which the MPR121 capacitive touch sensor arrays are read and, in the event of a touch or release event, MIDI messages sent over TTY serial to the HIDUINO-equipped communications microcontroller. As shown in the figure, the firmware is quite simple, and (following configuration of the MPR121 devices) simply loops through the incoming messages from the MPR121 devices, checking to see whether an electrode's current state is different than its previous state. If the state is different and the electrode is currently sensing a touch event, then a MIDI NoteOn message corresponding to the electrode's number is sent to the ATMEGA32U2; if the state is different and the electrode is not sensing a touch event, a MIDI NoteOff event is sent. After the ATMEGA32U4 receives the TTY serial message, it is converted into a USB MIDI HID event and is sent over USB to the host PC.

The host PC makes use of the Ableton Live digital audio workstation software to read incoming MIDI messages and adjust the gain of pertinent audio clips. As discussed in Section 3.2, an ambient drone plays continually in the background, attenuating only when an electrode touch event occurs. Therefore, any incoming MIDI NoteOn message attenuates the drone track and, concurrently, increases the gain on the audio clips whose electrodes were just touched. After one minute of no input events, the drone track begins to increase in gain, reaching a set volume and looping until a MIDI NoteOn event occurs.

\section{INSTALLATION AND USE}

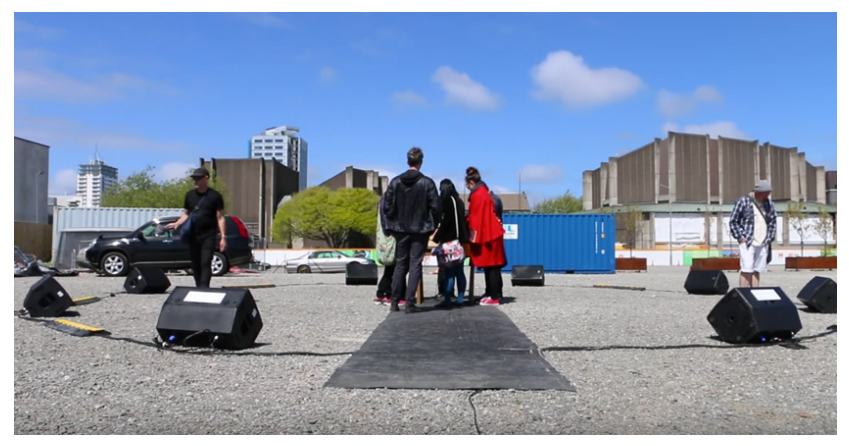

Figure 8: Lost Oscillations installed in Christchurch as part of the 2015 Audacious sound art festival. Visible here are a number of the installation's eight loudspeakers, arranged in a ring around the central user interface.

Lost Oscillations debuted at Christchurch's 2015 Audacious Festival of sonic art works. As a site-specific installation, the piece's location and on-site configuration are significant to the work's outcome. As such, these two elements are discussed in detail in the following two subsections.

\subsection{Location}

When installed, the user interface and speaker array were positioned outdoors in the center of Christchurch, surrounded by the locations at which the piece's sound materials were recorded or which are referred to in spoken-word materials. The virtual soundscape therefore has a strong situated audio aspect, using sound spatialization in combination with topographic cues provided by the stylized map and the location of the sensors on it, to alert users to the location of historical events and materials in terms of the actual soundscape around them. Furthermore, the ambiguity between the actual and virtual (8-channel) soundscapes createa strong mixedreality experience, thereby sonically and experientially connecting the past and present soundscapes of the city, and blending its virtual sounds with the present-day landscape of the city. It was intended that such blending would result in spatiotemporal ambiguity, requiring that participants determine whether a sound was created in the "here and now" of the city or, rather, in the "there and then" of the virtual sound world.

To couple with the vital materiality of the interface's physical components, a rock-strewn vacant lot in central Christchurch (shown in Figure 8) was selected as the installation location. As the work was sited at the location the Christchurch Art Gallery prior to its demolition in the aftermath of the 2010 Canterbury Earthquake, the vacant lot served as a tabula rasa (surrounded by the city and its ambient noises) from which the contemporary cityscape's sounds could be combined with user-triggered historical audio events.

\subsection{Configuration and Use}

After selecting a location, the on-site configuration of Lost Oscillations was considered. To allow for relatively accurate placement of phantom audio sources, an eight-channel loudspeaker array consisting of large weather-resistant monitor speakers was chosen as the means of audio playback. The loudspeakers are positioned around the centrally-located user interface: users approach the user interface by stepping inside the ring of loudspeakers, an act which physically and metaphorically immerses them in the situated mixed-reality audio-space of the piece's situated audio.

After placing the speakers, the individual audio files' gains were adjusted in situ, with the objective of balancing the audio files' loudness with that of the ambient noise of the Christchurch cityscape. After completing the level balancing, many of the field recordings were difficult to distinguish from real-world sounds occurring in the city at large; such ambiguity between audio file and ambient noise indicated that the pursuit of inconspicuous situated audio was a successful one.

Once the installation was opened to the public, participants began to engage with the artwork. It was observed that the multitouch and multi-user capabilities of the audio interface led to interesting and unanticipated interactions between different users. When more than one user was touching the interface's various electrodes, some participants began to explore different rhythmic and timbral means of interlocking the audio files that they each controlled. Such open-ended user interaction schemes indicate that the interface developed for Lost Oscillations is a flexible device that may be re-used or extended in future pieces with different contexts.

During the installation of Lost Oscillations, video footage was captured in order to allow the inherently 
transient site-specific work to be viewed by those unable to visit it. This documentation may be viewed at https://www.youtube.com/watch?v=eajBHvvfNUs.

\section{FUTURE WORK}

Lost Oscillations is the first in an intended series of interactive sound art installations focusing on the spatio-temporal exploration of sound. After installing and qualitatively evaluating Lost Oscillations, a number of aspects will be altered in future iterations of the piece.

The electrodes in Lost Oscillations are fixed in space, attached to the surface of the interface's enclosure. While touching the electrodes results in spatially relevant audio, there is no accompanying physical analog to the temporality of the audio. Future versions of the interface will feature electrodes mounted to sensors that allow vertical displacement to be transduced, letting users press down to explore "deeper," older sounds. Such depth-related control over the sound will further explore the underlying theme of sonic archeology, allowing users to metaphorically excavate a certain area's sound, beginning by exploring recent events and, by pressing down and lowering the electrode, proceeding through to listen to older sounds. Additionally, the depth element is envisaged as increasing the affective affordance of the interface, as interaction requires increased effort and control from users, encouraging them to invest greater attention to, and corporeal engagement with, the installation.

After exploring enhancements to the transducers, additional versions of Lost Oscillations will be developed, each one pertaining to a different city or region. A further area for development in these regionally-discrete interfaces will be the employment of regionally relevant materials in the construction of their interfaces, further coupling an area's physical materiality with the sounds that are explored by those using the interface.

\section{CONCLUSIONS}

As an interactive auditory installation, Lost Oscillations combines technical and aesthetic sonic arts subdiscplines in order to create a piece that requires the human touch to explore the contemporary and historical soundscapes of Christchurch, New Zealand.

Technically, Lost Oscillations serves as a case study in user interface design for interactive auditory artworks, demonstrating that a relatively simple, low-parts-count, driverless interface may be effectively used to allow participants a meaningful way to interact with situated audio. It is hoped that the design and development of the physical interface for Lost Oscillations may serve as a starting point for future auditory installation interfaces.
Aesthetically and conceptually, the outcome of Lost Oscillations may be viewed as a success: participants were able to engage in a physical and aural exploration of the "sonic archeology" of the city, experiencing the city's auditory past within the greater context of the present-day soundscape of the city. It is anticipated that the technical, conceptual, and aesthetic outcomes of Lost Oscillations will be the first in a series of related artworks focusing on the layered spatio-temporal sonic stratigraphy of cities and other spaces.

\section{ACKNOWLEDGMENTS}

(Section Removed for Anonymous Review Process)

\section{REFERENCES}

[1] (Reference Removed for Anonymous Review Process)

[2] Johnson, B. (2014). Emerging Technologies for Real-Time Diffusion Performance. In Leonardo Music Journal, Volume 24. MIT Press.

[3] (Reference Removed for Anonymous Review Process)

[4] Park, T. H, et al. (2013). Locative Sonification: Playing the World Through Citygram In Proceedings of The 2013 International Computer Music Conference (ICMC-2013), Perth, Australia.

[5] Bennett, J (2010). Vibrant Matter: A Political Ecology of Things, Duke University Press.

[6] NXP Semiconductors N.V. (2014). I I C Bus Specification and User Manual, NXP Semiconductors,.

[7] Diakopoulos, D and Kapur, A. (2011). HIDUINO: A firmware for building driverless USB-MIDI devices using the Arduino microcontroller In Proceedings of the 2011 Conference on New Interfaces for Musical Expression, Oslo, Norway. 